Notfall Rettungsmed 2016 $19: 249$

DOI 10.1007/s10049-016-0183-4

Online publiziert: 23. Mai 2016

๑) Springer-Verlag Berlin Heidelberg 2016

CrossMark

\section{G. Rücker}

Klinik und Poliklinik für Anästhesiologie und Intensivtherapie, Universitätsmedizin Rostock, Rostock, Deutschland

\section{Fieber}

Liebe Leserinnen und Leser,

dank der weitreichenden Akzeptanz von Leitlinien ist das technische Monitoring in der präklinischen Notfallmedizin nicht mehr wegzudenken. Leise im Hintergrund hat sich im Zuge der Innovation von Sensoren nunmehr ein zusätzlicher Parameter als Standard durchgesetzt: die Messung der Körpertemperatur. Fast jedes Rettungsmittel verfügt daher heute über ein Infrarotthermometer. Als sich diese Messmethode zu etablieren begann, hatten wir im Rahmen der Fragestellung, ob routinemäßig die Körpertemperatur miterfasst werden sollte, an unserem Notarztstützpunkt Warnow Ost im Landkreis Rostock diesen Parameter für eine definierte Zeit erhoben. Immerhin $8 \%$ der Einsätze $(n=358$, nicht publiziert) wiesen dort eine Abweichung vom Normalwert auf. Nicht nur die unerkannte Hypothermie, sondern auch die nicht erwartete Temperaturerhöhung gerieten damit zunehmend in den Fokus differenzialdiagnostischer Erwägungen im Rettungsdienst. Die Erhöhung der Temperatur über den Normalwert hinaus, im Allgemeinen als Fieber bezeichnet, ist ein wichtiges Symptom für eine große Anzahl von Erkrankungen aus den unterschiedlichsten Fachdisziplinen und bisweilen sogar Leitsymptom.
Das vorliegende Leitthemenheft „Fieber" beleuchtet vier Facetten dieses wichtigen Symptoms. Im Beitrag von Böhmer et al. wird der allgemeinmedizinische Aspekt behandelt. Der Erkenntnisgewinn aus dieser Perspektive ist insofern von Bedeutung, da diese für den Rettungsdienst wichtige allgemeinmedizinische Schnittstelle einen Großteil der Patienten abfiltert und in der Folge kritische Verläufe hier einen respektablen Anteil der eingewiesen Patienten darstellen. Zahlreiche neurologische Erkrankungen hingegen zeigen ebenfalls Verläufe mit Fieber, die im Artikel von Wittstock et al. abgehandelt werden. Selbstverständlich treten auch eine große Anzahl von Tropenerkrankungen durch ausgedehnte Reisetätigkeit, globale Migrationsbewegungen oder interkontinentale Geschäftsbeziehungen auf, die im Artikel von Hemmer et al. aufgezeigt werden. Deren aller Diagnostik wird bei entsprechender Risikokonstellation durch eine mikrobiologische Untersuchung ergänzt, für die spezielle Entnahme- und Transportbedingungen eingehalten werden müssen. Einen Einblick hierüber soll abschließend der Artikel von Devide liefern.

Selbstverständlich kann das Symptom Fieber im Rahmen eines einzelnen Hefts nicht allumfassend abgehandelt werden. Dafür sind die Krankheitsursachen zu zahlreich. Deshalb planen wir, diesen Fokus mit dem Leitthemenheft „Infektiologie und Hygiene bei Notfallpatienten“ in der Ausgabe 1/2017 weiterzuführen.

Wir hoffen, dass wir Ihnen mit diesem Heft einen kleinen Überblick über das große Feld der fiebrigen Erkrankungen geben können und wünschen Ihnen viel Spaß und Erkenntnisgewinn bei der Lektüre.

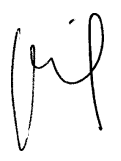

Gernot Rücker

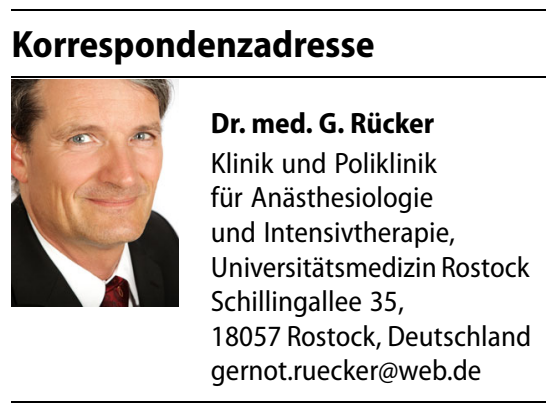

Interessenkonflikt. G. Rücker gibt an, dass kein Interessenkonflikt besteht. 